\title{
Oral Cancer with Verrucous Pattern is Not Associated with Human Papilloma Virus in Indian Population
}

\begin{abstract}
Background: The etiology of verrucous lesions of the oral cavity is debatable, and many western studies attribute it to human papillomavirus (HPV) infection. Although most Indian studies have found a strong association with tobacco chewing, the role of HPV has not been studied in the Indian context. Materials and Methods: A prospective study was conducted on the clinicopathological profile of 21 consecutive patients of verrucous lesions of the oral cavity. The patients were evaluated on the basis of addictions, pretreatment biopsy, p16 immunohistochemistry (IHC), and histopathological parameters. Results: Preoperative incisional biopsy revealed no dysplasia in $52.38 \%$, mild-to-moderate dysplasia in $19.04 \%$, and invasive carcinoma in $28.57 \%$ of the patients. About $67 \%$ of patients underwent surgical excision in our institute all of whom had invasive malignancy on final histopathology. This included $42.85 \%$ patients whose initial biopsy was no or mild dysplasia. All of the patients were negative for p16 IHC. Conclusions: Verrucous lesions in Indian population are caused by smokeless tobacco unlike that in the western countries where HPV is the main etiology. Clinical distinction between benign and malignant lesions is difficult, and we recommend complete surgical excision of the lesion with adequate margins whenever possible.
\end{abstract}

Keywords: Human papillomavirus, oral cavity and human papillomavirus, p16 immunohistochemistry, smokeless tobacco, verrucous lesions

\section{Introduction}

Verrucous lesions of the oral cavity comprise of a varied set of disorders such as verrucous hyperplasia, proliferative verrucous leukoplakia, and verrucous carcinoma. The etiology of these lesions is still under debate, and there are conflicting results in the literature regarding its etiology. There is plethora of reports from the western world attributing Human Papillomavirus (HPV) infection to verrucous lesions. It is well known that a "benign looking" verrucous lesion can harbor invasive malignancy. We investigated the role of HPV in the etiology of the verrucous lesions of the oral cavity and analyzed their clinicopathological profile.

\section{Materials and Methods}

We prospectively studied the clinicopathological profile including the p16 immunohistochemistry (IHC) assays (a surrogate marker for HPV infection) of 21 consecutive patients of verrucous lesions of the oral cavity

This is an open access journal, and articles are distributed under the terms of the Creative Commons Attribution-NonCommercial-ShareAlike 4.0 License, which allows others to remix, tweak, and build upon the work non-commercially, as long as appropriate credit is given and the new creations are licensed under the identical terms.

For reprints contact: reprints@medknow.com presenting in our institute [Table 1]. The patients were evaluated on the basis of addictions, pretreatment biopsy, and p16 IHC. Correlation with histopathological parameters was done for those patients who underwent surgical excision of the lesions at our institution [Table 2].

Histopathological diagnosis of verrucous lesions was confirmed microscopically on hematoxylin and eosin $(\mathrm{H}$ and $\mathrm{E})$ stained sections. IHC was performed on all the cases using Roche p16 CINtec Histology kit ${ }^{\circledR}$ with 1: 10 dilution, heat-induced antigen retrieval and DAB (3,3-diaminobenzidine) as chromogen. The cases were reported as per the guidelines provided by the Roche p16 CINtec Histology kit $^{\circledR}$. Moderate-to-strong nuclear or both nuclei and cytoplasm positivity in more than or at least $70 \%$ tumor cells (but not cytoplasmic positivity alone) was considered positive. Cases exhibiting weak immunoreaction were considered negative [Figure 1].

As the p16IHC assay was an additional test performed on the routine histopathology specimen, it did not require any added intervention to the patient. The results

How to cite this article: Chakrabarti S, Banerjee D,
Qayyumi BN, Nair D, Nair S, Kane S, et al. Oral cancer
with verrucous pattern is not associated with human
papilloma virus in Indian population. Indian J Med
Paediatr Oncol 2018;39:479-82.

\section{Swagnik \\ Chakrabarti, Devmalya Banerjee ${ }^{1}$, Burhanuddin Nuruddin Qayyumi, Deepa Nair, Sudhir Nair, Subhada Kane ${ }^{1}$, Pankaj Chaturvedi}

Departments of Surgical Oncology and ${ }^{1}$ Pathology, Tata Memorial Hospital, Mumbai, Maharashtra, India

Address for correspondence: Prof. Pankaj Chaturvedi, Department of Surgical Oncology, Tata Memorial Hospital, Dr. E Borges Road, Parel, Mumbai - 400 011, Maharashtra, India. E-mail: chaturvedi.pankaj@ gmail.com 


\begin{tabular}{|c|c|c|c|c|c|c|}
\hline \multicolumn{7}{|c|}{ Table 1: Patient demographics } \\
\hline Patient serial number & Age (years) & Gender & Habits & Subsite & Pretreatment biopsy & p16 IHC \\
\hline 1 & 54 & Male & Tobacco chewing & Floor of mouth & Invasive SCC & Negative \\
\hline 2 & 43 & Male & Smoking, alcohol and tobacco chewing & Retromolar trigone & Moderate dysplasia & Negative \\
\hline 3 & 62 & Male & Tobacco chewing & Buccal mucosa & No dysplasia & Negative \\
\hline 4 & 38 & Male & Tobacco chewing & Buccal mucosa & No dysplasia & Negative \\
\hline 5 & 69 & Male & Tobacco chewing & Buccal mucosa & No dysplasia & Negative \\
\hline 6 & 54 & Male & Tobacco chewing & Buccal mucosa & No dysplasia & Negative \\
\hline 7 & 64 & Male & Tobacco chewing & Lower alveolus & Moderate dysplasia & Negative \\
\hline 8 & 55 & Male & Gutka and tobacco chewing & Tongue & Mild dysplasia & Negative \\
\hline 9 & 48 & Male & Tobacco chewing & Buccal mucosa & Moderate dysplasia & Negative \\
\hline 10 & 78 & Male & Tobacco chewing & Lower alveolus & No dysplasia & Negative \\
\hline 11 & 56 & Female & Masher & Buccal mucosa & No dysplasia & Negative \\
\hline 12 & 61 & Male & Smoking and tobacco chewing & Buccal mucosa & Invasive SCC & Negative \\
\hline 13 & 48 & Male & Tobacco chewing & Tongue & Invasive SCC & Negative \\
\hline 14 & 65 & Male & Smoking, alcohol and tobacco chewing & Buccal mucosa & Invasive SCC & Negative \\
\hline 15 & 26 & Male & Smoking, alcohol and tobacco chewing & Buccal mucosa & No dysplasia & Negative \\
\hline 16 & 44 & Male & Tobacco chewing & Buccal mucosa & No dysplasia & Negative \\
\hline 17 & 45 & Male & Tobacco chewing & Buccal mucosa & No dysplasia & Negative \\
\hline 18 & 45 & Male & Tobacco chewing & Buccal mucosa & No dysplasia & Negative \\
\hline 19 & 58 & Female & Tobacco chewing & Tongue & Verrucous ca & Negative \\
\hline 20 & 60 & Female & Tobacco chewing & Lower alveolus & Invasive SCC & Negative \\
\hline 21 & 45 & Male & Tobacco chewing & Buccal mucosa & No dysplasia & Negative \\
\hline
\end{tabular}

IHC - Immunohistochemistry; SCC - Squamous cell carcinoma

Table 2: Pathological profile of the patients who underwent surgical excision of the lesion

\begin{tabular}{|c|c|c|c|c|c|c|c|}
\hline Patient serial number & Postsurgery histopathology & Differentiation & "pT" stage & Depth of invasion $(\mathrm{cm})$ & PNI & LVS & "pN" stage \\
\hline 1 & Invasive SCC & Moderately differentiated & T1 & 0.3 & Nil & Nil & N0 \\
\hline 2 & Invasive SCC & Well differentiated & $\mathrm{T} 2$ & 0.5 & Nil & Nil & No \\
\hline 3 & Invasive SCC & Well differentiated & $\mathrm{T} 2$ & 0.2 & Nil & Nil & NA \\
\hline 4 & Invasive SCC & Well differentiated & $\mathrm{T} 2$ & 0.2 & Nil & Nil & N0 \\
\hline 5 & Invasive $\mathrm{SCC}$ & Well differentiated & $\mathrm{T} 1$ & 0.3 & Nil & Nil & N0 \\
\hline 6 & Invasive SCC & Well differentiated & $\mathrm{T} 2$ & 0.2 & Nil & Nil & N0 \\
\hline 7 & Invasive SCC & Moderately differentiated & $\mathrm{T} 4$ & 1.8 & Nil & Nil & No \\
\hline 8 & Invasive $\mathrm{SCC}$ & Well differentiated & $\mathrm{T} 3$ & 0.4 & Nil & Nil & NA \\
\hline 9 & Invasive SCC & Well differentiated & $\mathrm{T} 1$ & 0.8 & Nil & Nil & NA \\
\hline 12 & Invasive $\mathrm{SCC}$ & Well differentiated & $\mathrm{T} 2$ & 1.5 & Nil & Nil & No \\
\hline 13 & Invasive $\mathrm{SCC}$ & Well differentiated & $\mathrm{T} 2$ & 1.5 & Nil & Nil & N0 \\
\hline 19 & Invasive SCC & Moderately differentiated & $\mathrm{T} 2$ & 0.8 & Nil & Nil & No \\
\hline 20 & Invasive $\mathrm{SCC}$ & Well differentiated & $\mathrm{T} 1$ & 0.3 & Nil & Nil & No \\
\hline 21 & Invasive SCC & Verrucous ca & $\mathrm{T} 2$ & 0.4 & Nil & Nil & N0 \\
\hline
\end{tabular}

NA - Patients in whom neck dissection was not done; SCC - Squamous cell carcinoma; PNI - Perineural Invasion; LVS - Lympho Vascular spread

were not expected to have any impact on the physical, functional, emotional, social, or management aspect of the patient. As per our hospital policy, an ethical approval from the Institutional Review Board for such a study was not required.

\section{Results}

Table 1 depicts the patient demographics. Majority of the patients were males (18 males and 3 females) and all were smokeless tobacco users. The mean age was 53.23 years (range 26-78 years). Thirteen patients had lesions in the buccal mucosa [Figure 2], 3 on the lower alveolus, 3 on oral tongue [Figure 3], and 1 each in the retromolar trigone and floor of the mouth. Incisional biopsy was taken from the most suspicious area. The biopsy revealed no evidence of dysplasia in 11, mild dysplasia in 1 , moderate dysplasia in 3, and invasive squamous cell carcinoma (SCC) in 6 patients. Fourteen out of twenty-one patients underwent surgery at our institution. Six patients were kept in close follow-up on the basis of no evidence of dysplasia on biopsy. One patient having invasive malignancy on biopsy preferred to undergo surgery at his native place. All the patients who underwent surgery (14 patients) in our institute were reported to have invasive carcinoma in 


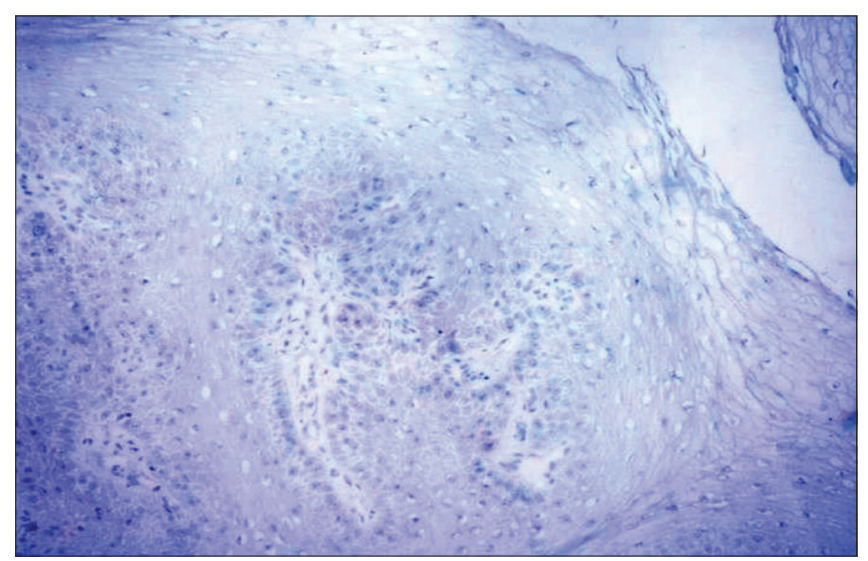

Figure 1: p16-negative immunohistochemistry in a case of verrucous carcinoma of oral cavity $(\times 200)$

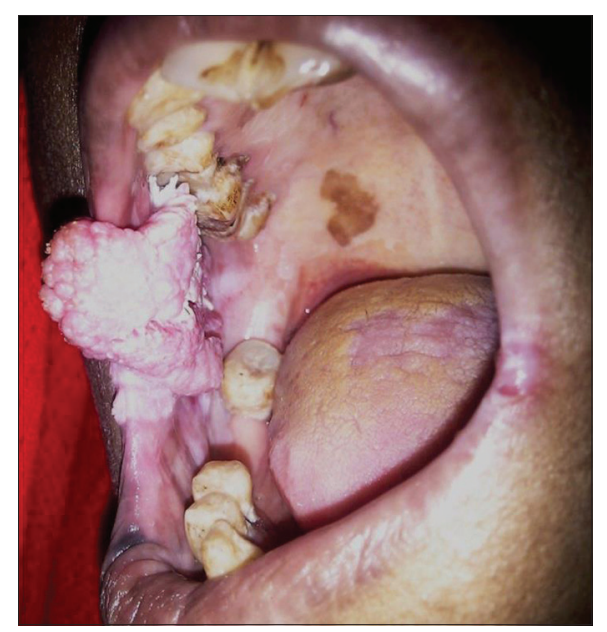

Figure 2: Verrucous lesion buccal mucosa

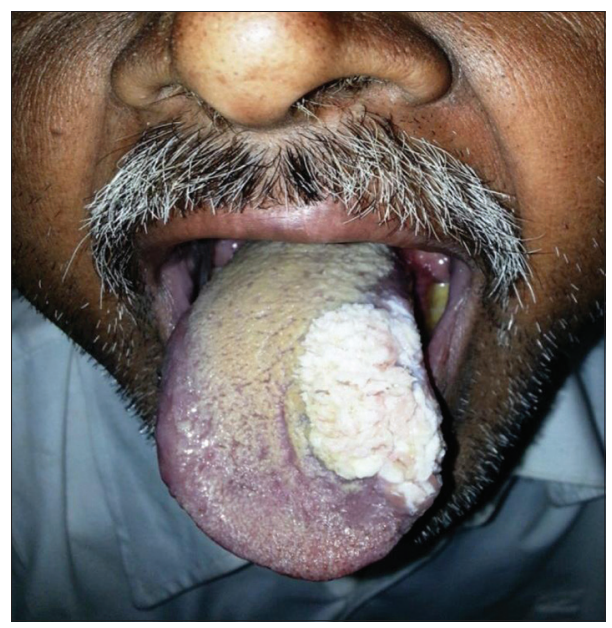

Figure 3: Verrucous lesion tongue

final histopathology report [Table 2]. Of these 10 had well differentiated, 3 had moderately differentiated squamous carcinoma and 1 verrucous carcinoma. The majority of the patients (12/14) were either T1 or T2 lesions. Eleven patients underwent elective neck dissection all of whom were found to be negative for neck node metastasis. The mean depth of invasion was $0.72 \mathrm{cms}$ (range $0.2-1.8 \mathrm{~cm}$ ) with only 4 patients having the depth of invasion more than $1 \mathrm{cms}$. None of the patients had perineural invasion or lymphovascular spread. All the resected specimens were found negative for p16 IHC [Figure 1].

\section{Discussion}

Verrucous lesions of the oral cavity comprise of a wide spectrum of clinicopathologically overlapping lesions. Verrucous hyperplasia was first described by Shear and Pindborg. ${ }^{[1]}$ It is a benign lesion, clinically indistinguishable from verrucous carcinoma. Verrucous leukoplakia is a thick white keratinized lesion having an exophytic growth pattern. ${ }^{[2]}$ One of its variants, the proliferative verrucous leukoplakia (PVL) presents as multifocal lesion affecting elderly people and carries a high malignant transformation potential. ${ }^{[3,4]}$ Verrucous carcinoma is an uncommon tumor first described by Ackerman. ${ }^{[5]}$ It is a form of well-differentiated squamous cell carcinoma with specific clinical and histological factors. The tumor is a slow growing, locally invasive, proliferative, painless plaque-like lesion that usually does not metastasize to regional neck nodes.

There has been significant controversy in literature regarding the etiology of verrucous lesions. Some authors have emphatically attributed warty lesions of the oral cavity including benign lesions, PVL, and verrucous carcinoma to HPV. ${ }^{[6,7]}$ Fornatora identified a specific variant of oral epithelial dysplasia, koilocytic dysplasia, which harbored features of HPV infection on light microscopy (Fornatora et al., 1996). ${ }^{[8]}$ In a series of 139 patients, HPV DNA was found in $17.6 \%$ of patients of oral leukoplakia and $19.7 \%$ patients of oral lichen planus as compared to $5.6 \%$ of controls. ${ }^{[9]}$

In a series of 9 lesions of PVL, HPV infection was found in $89 \%$ of patients. ${ }^{[10]}$ In all the patients, HPV DNA was assessed using PCR technique in biopsy specimens. However, in another series of 13 patients, HPV infection could not be detected in any of the PVL lesions. ${ }^{[11]}$ In this study, HPV DNA was assessed using PCR technique in biopsy specimens of 10 patients and oral rinse specimens of 4 patients. Patients in all stages of the disease ranging from simple hyperkeratosis to oral SCC were included in the study.

Although most western studies relate verrucous lesions to HPV, none of the patients in our study had evidence of HPV infection in their biopsy specimens. Interestingly, all patients were smokeless tobacco users and 4 had multiple addictions. This study is supported by another study of 133 patients from the Indian subcontinent which showed a strong association of tobacco chewing with verrucous carcinoma. ${ }^{[12]}$ Another Indian study assessed the clinicopathological profile of patients with verrucous hyperplasia (patients with verrucous carcinoma were 
excluded) ${ }^{[13]}$ In the 19 patients studied, there was a strong association of tobacco and lime quid placement in the buccal mucosa with verrucous hyperplasia.

Although most studies from India have found an association of tobacco chewing with verrucous lesions, none have investigated the association of these lesions with HPV. This study shows that verrucous lesions in the Indian subcontinent are not associated with HPV infection unlike that in the western countries. This may be due to the different pathogenesis of the disease process in the Indian population where there is high consumption of tobacco in the chewable form.

Diagnosis of verrucous lesions is challenging for clinicians as well as pathologists as they include a spectrum of benign, potentially malignant, and frankly invasive malignancies. The lesions have similar gross morphology and distinguishing them clinically is difficult, even an incisional biopsy may be misleading. There is a lot of confusion regarding the current clinical and histopathological guidelines to diagnose this potentially malignant entity. ${ }^{[14]}$ The most reliable way to separate these entities on routine $\mathrm{H}$ and $\mathrm{E}$ stained tissue sections is to recognize the exophytic growth patterns of oral verrucous hyperplasia from the combined exophytic and endophytic growth patterns associated with verrucous carcinoma. In our study, it was surprising that 6 patients had no dysplasia or mild dysplasia in the incisional biopsy specimen all of which were reported to harbor invasive carcinomas on final histopathology. This finding shows that most of these verrucous lesions are in fact invasive carcinoma at the time of presentation. This can be attributed to inadequate sampling or improper targeting of the lesion despite repeated biopsies. As all operated patients in our study harbored invasive malignancy, and repeated biopsies failed to detect underlying malignancy, we recommend a complete surgical excision of the lesion with a pathological three dimensional clear margin of $\geq 5 \mathrm{~mm}$. Furthermore, most of these carcinomas were early stage (12 of 14 operated patients had pT1 and pT2 lesions), and none had pathological adverse factors such as perineural invasion or lymphovascular spread. None of the 11 patients who underwent neck dissection had nodal metastasis. This emphasizes that complete surgical excision of these indolent lesions carries a good prognosis.

The study was conducted on a small number of lesions and we need to validate these findings in larger data set. Moreover, the HPV assay was done using p16 IHC which is a surrogate marker of HPV infection. Analysis using HPV DNA would have been more appropriate.

\section{Conclusions}

Oral verrucous lesions comprise of a diverse spectrum of diseases. Most of these lesions in Indian population are caused by smokeless tobacco unlike that in the western countries where HPV is the etiology. Differentiating the benign ones from the potentially malignant and frankly malignant ones based on clinical features or biopsy can be deceptive. We recommend a complete surgical excision with adequate margins for proper diagnosis and management.

\section{Acknowledgments}

We would like to thank Dr. Nikita Oza, Senior Resident, Dept. of Pathology, Tata Memorial Hospital, Mumbai for providing the microscopic photographs.

Financial support and sponsorship

Nil.

\section{Conflicts of interest}

There are no conflicts of interest.

\section{References}

1. Shear M, Pindborg JJ. Verrucous hyperplasia of the oral mucosa. Cancer 1980;46:1855-62.

2. Greenberg MS, Glick M. Burket's Oral Medicine Diagnosis \& Treatment. $10^{\text {th }}$ ed. Hamilton, Ontario: BC Decker; 2003. p. $87-105$.

3. Cabay RJ, Morton TH Jr., Epstein JB. Proliferative verrucous leukoplakia and its progression to oral carcinoma: A review of the literature. J Oral Pathol Med 2007;36:255-61.

4. van der Waal I, Reichart PA. Oral proliferative verrucous leukoplakia revisited. Oral Oncol 2008;44:719-21.

5. Ackerman LV. Verrucous carcinoma of the oral cavity. Surgery 1948;23:670-8.

6. Eversole LR. Papillary lesions of the oral cavity: Relationship to human papillomaviruses. J Calif Dent Assoc 2000;28:922-7.

7. Pringle GA. The role of human papillomavirus in oral disease. Dent Clin North Am 2014;58:385-99.

8. Fornatora M, Jones AC, Kerpel S, Freedman P. Human papillomavirus-associated oral epithelial dysplasia (koilocytic dysplasia): An entity of unknown biologic potential. Oral Surg Oral Med Oral Pathol Oral Radiol Endod 1996;82:47-56.

9. Campisi G, Giovannelli L, Aricò P, Lama A, Di Liberto C, Ammatuna P, et al. HPV DNA in clinically different variants of oral leukoplakia and lichen planus. Oral Surg Oral Med Oral Pathol Oral Radiol Endod 2004;98:705-11.

10. Palefsky JM, Silverman S Jr., Abdel-Salaam M, Daniels TE, Greenspan JS. Association between proliferative verrucous leukoplakia and infection with human papillomavirus type 16 . J Oral Pathol Med 1995;24:193-7.

11. Bagan JV, Jimenez Y, Murillo J, Gavaldá C, Poveda R, Scully C, et al. Lack of association between proliferative verrucous leukoplakia and human papillomavirus infection. J Oral Maxillofac Surg 2007;65:46-9.

12. Rekha KP, Angadi PV. Verrucous carcinoma of the oral cavity: A clinico-pathologic appraisal of 133 cases in Indians. Oral Maxillofac Surg 2010;14:211-8.

13. Hazarey VK, Ganvir SM, Bodhade AS. Verrucous hyperplasia: A clinico-pathological study. J Oral Maxillofac Pathol 2011;15:187-91.

14. Kallarakkal TG, Ramanathan A, Zain RB. Verrucous papillary lesions: Dilemmas in diagnosis and terminology. Int $\mathrm{J}$ Dent 2013;2013:298249. 